\title{
Kindergartenkinder als Zeugen von Mobbing
}

\section{Debora Hauser, Eveline Gutzwiller-Helfenfinger und Françoise D. Alsaker}

In der vorliegenden Studie wurde untersucht, wie Kinder reagieren, wenn sie Zeugen von Mobbing werden. 968 Kinder aus 59 Kindergärten, in denen Mobbing stattfand, nahmen an der Studie teil. Es zeigten sich Alters- und Geschlechtseffekte bei Mobber-und Opfer-unterstützendem Zeugenverhalten sowie beim Ausmass an hilflosen Reaktionen. Zudem variierte das Zeugenverhalten in Abhängigkeit von der Mobbingrolle: Mobber zeigten mehr Mobber-unterstützende, nicht-involvierte Kinder mehr Opfer-unterstützende und passive Opfer mehr hilflose Reaktionen. Auch das habituelle Verhalten der Kinder in der Gruppe hing mit den Zeugenreaktionen der einzelnen Kindern zusammen: Viel aggressives Verhalten in der Kindergartengruppe ging mit mehr Mobber-unterstützenden Reaktionen einher, während es mehr Opfer-unterstützende Reaktionen in Gruppen gab, in welchen Konfliktsituationen eher positiv gehandhabt wurden. Die Rolle der Zeugen wird im Rahmen präventiver Ansätze diskutiert.

Mobbing im Kindergarten wird erst seit wenigen Jahren erforscht, wobei bislang vor allem die Charakteristika und das Verhalten der direkt involvierten Kinder, d.h. der Mobber und ihrer Opfer, im Vergleich zu den nicht-involvierten Kindern im Zentrum des Interesses standen (Hawker \& Boulton, 2000; Perren \& Alsaker, 2006). Wenig ist jedoch darüber bekannt, wie Kinder auf der emotionalen und der Verhaltensebene reagieren, wenn sie Zeugen von Mobbing werden, das heisst, wenn sie beobachten, dass andere Kinder mobben oder gemobbt werden. Kinder, die bei Mobbingvorfällen zwar anwesend sind, aber weder die Rolle des Täters noch die des Opfers einnehmen, werden im Folgenden Mobbingzeugen genannt.

Anders als in bisherigen Studien (z.B. Salmivalli, Lagerspetz, Björkquvist, Österman \& Kaukiainen, 1996) betrachten wir die Zeugenreaktionen der Kinder als variable Verhaltensmerkmale und definieren keine Verhaltenskategorien: Es werden demnach keine feste Rollen als Mobbingzeugen gebildet. Methodisch bedeutet es, dass die Kinder einen Wert für jede der aktuellen Verhaltensvaria- 
blen erhalten. Damit wird auf theoretischer Ebene situationalen Gegebenheiten sowie Gruppenprozessen Rechnung getragen, da wir davon ausgehen, dass die Kinder sich in unterschiedlichen Situationen (mit teils unterschiedlichen Akteur/innen) auch als Zeugen unterschiedlich verhalten. Dieses Vorgehen entspricht einem eher state- als trait-orientierten Zugang zum Zeugenverhalten.

Diese Vorgehensweise ist weiter wichtig, da auch Mobber und Opfer Mobbingzeugen sein können, nämlich dann, wenn ein anderes Kind als sie selber mobbt oder gemobbt wird. Mobbingzeugen sind nicht nur Beobachter des Geschehens, sie können auch zugunsten des Opfers oder des Mobbers ins Geschehen eingreifen. In bisherigen Studien wurde zwischen zwei bis vier verschiedenen Zeugenreaktionen unterschieden, die je nach Autoren anders benannt wurden, wie z.B. adäquate, inadäquate und hilflose Zeugenreaktionen (Gutzwiller-Helfenfinger \& Alsaker, 2005, 2007); aggressive Reaktionen, prosoziale Reaktionen und Rückzugsverhalten (Lindeman, Harakka \& Keltikangas-Järvinen, 1997); sozial angemessene und sozial unangemessene Zeugenreaktionen (Craig $\&$ Pepler, 1997); oder auch passive, aktive und negative Zuschauer (Staub, Fellner, Berry \& Morange, 2003). In der vorliegenden Studie wurden drei voneinander unterscheidbare Kategorien von Zeugenreaktionen gebildet, welche die Inhalte der soeben genannten Kategorien aus der Literatur zusammenfassen. Es sind: Mobber-unterstützende, Opfer-unterstützende und hilflose Zeugenreaktionen.

Beobachtungsstudien aus Kanada konnten zeigen, dass Peers in 85 bis $88 \%$ der Mobbingvorfälle Zeugen der Geschehnisse sind und dass sie durch ihr Verhalten die Dauer und den Ausgang der Vorfälle beeinflussen (Atlas \& Pepler, 1998; Craig \& Pepler, 1995, 1997; Hawkins, Pepler \& Craig, 2001; O’Connell, Pepler \& Craig, 1999). Mobbingzeugen intervenieren allerdings nur in $10 \% \mathrm{der}$ Vorfälle im Klassenzimmer (Atlas \& Pepler, 1998) und in $11 \%$ der Vorfälle auf dem Pausenplatz (Craig \& Pepler, 1995, 1997). Hawkins et al. (2001) berichten von Mobbingzeugen, die in $88 \%$ der Mobbingvorfälle anwesend waren und in lediglich 19 \% dieser Vorfälle intervenierten. Sowohl Rigby und Slee (1991) als auch Menesini, Eslea, Smith, Genta, Giannetti, Fonzi und Costabile (1997) berichten, dass die Mehrheit der befragten Schüler dem Opfer gegenüber positiv und dem Mobber gegenüber negativ eingestellt sind. Diese verschiedenen Befunde zeigen, dass möglicherweise auch unter den Mobbingzeugen - und nicht nur unter den Opfern - Hilflosigkeit herrscht und nur wenige den Mut haben zu intervenieren. Es werden allerdings auch Geschlechtsunterschiede in den Einstellungen gegenüber Mobbing berichtet. So äussern Jungen weniger Sympathie den Opfern gegenüber als Mädchen. Diese geben auch an, betroffener zu sein als die Jungen, und sie regen sich mehr über das Mobbing auf (Menesini et al., 1997; Rigby \& Slee, 1991; Schäfer, 1996). Wenn die Mehrheit der Kinder eine ablehnende Haltung gegenüber Mobbing hat, jedoch selten zugunsten der Opfer interveniert, stellt sich die Frage, wie diese Diskrepanz zwischen Einstellung und Verhalten zustande kommt. Die persönliche Intention, dem Opfer zu hel- 
fen, scheitert möglicherweise an den in der Peergruppe ablaufenden Mechanismen wie Gruppendruck oder soziale Ansteckung etc. So kann es sein, dass sich neben den Mobbern noch weitere, gewöhnlich nicht aggressive Kinder aktiv an den Taten beteiligen, anstatt dem Opfer zu helfen. Ein aus der sozialpsychologischen Forschung bekannter Gruppenmechanismus ist das Phänomen der Verantwortungsdiffusion, welches mit einem abgeschwächten Gefühl individueller Verantwortlichkeit einhergeht. Eine einzelne Person kann das Gefühl ihrer eigenen Verantwortlichkeit für eine negative Handlung (z.B. ein Kind auslachen) als erheblich vermindert empfinden, wenn mehrere Personen daran teilnehmen (Olweus, 2002). Diese Verantwortungsdiffusion führt dazu, dass die Schuldgefühle des Einzelnen nach dem Zwischenfall geringer sind und die Wahrscheinlichkeit von möglichen negativen Konsequenzen (z.B. Bestrafung) als tief eingeschätzt wird, weil viele Andere auch mitgemacht haben (Alsaker, 2003; Olweus, 2002). Weiter zeigen Studien mit Erwachsenen, dass mit steigender Anzahl Zeugen deren Bereitschaft zu helfen signifikant abnimmt (Darley \& Latané, 1968; Latané \& Darley, 1968). Wir nehmen zudem an, dass die Reaktionen der Mobbingzeugen entscheidend durch Modelllernen beeinflusst werden. Ein Modell wird eher imitiert, wenn es eine starke und einflussreiche Figur ist, für sein Verhalten belohnt und nicht bestraft wird und wenn es ähnliche Charakteristika mit dem Beobachter teilt (Bandura, 1977). In Bezug auf Mobbing sind zwei dieser Bedingungen meistens erfüllt. Die Mobber sind einflussreiche Figuren, die selten für ihr Verhalten bestraft werden. Aus den Beobachtungsstudien von Craig und Pepler $(1995,1997)$ geht nicht nur hervor, dass die Peers in lediglich $11 \%$ der Mobbingsituationen intervenierten, sondern auch dass die Lehrpersonen in nur $4 \%$ der Mobbingsituationen eingriffen. In 80\% der Mobbingepisoden waren sich die Lehrpersonen gar nicht erst bewusst, dass Mobbing stattfand. Die Tatsache, dass die Mobber nur in sehr begrenztem Ausmass von Seiten der Lehrkräfte, Eltern oder Gleichaltrigen bestraft (Craig \& Pepler, 1995, 1997; Olweus, 2002) und im Gegenzug dazu sogar mit ihrem Sieg über das Opfer belohnt bzw. verstärkt werden, kann dazu führen, dass die natürliche Aggressionshemmung bei den Mobbingzeugen geschwächt wird. Olweus (2002) nennt diesen Mechanismus das «Nachlassen der Hemmung gegen aggressive Tendenzen». Durch ihre Modellfunktion scheinen die Mobber in der Lage zu sein, ihre sonst «neutralen» Peers so zu beeinflussen, dass diese selbst aktiv beim Mobbing mitwirken. Jene Zeugen, die nicht aktiv beim Mobbing mitmachen, geben an, dass sie das Mobbinggeschehen nicht angehe oder dass sie Angst haben, selbst Opfer zu werden (Slee, 1993). Diese Passivität wird von den Mobbern häufig als Billigung ihres eigenen Verhaltens interpretiert und wirkt so eindeutig verstärkend (Alsaker, 2003). Erfreulich ist, dass die Mehrheit der Interventionen von Mobbingzeugen (57\%) zu einem Abbruch des Mobbingvorfalls führte.

Um Mobbing als Gruppenphänomen zu erfassen, haben Salmivalli und Kollegen (1996) den «Participant Role Ansatz» entwickelt. Ihr Ausgangspunkt war gerade die Annahme, dass neben den Mobbern und Opfern auch andere Kinder 
und Jugendliche in den Mobbingprozess involviert sind. Sie konnten anhand von Peer-Ratings 87 \% der 12- bis 13-jährigen Schülerinnen und Schüler in ihrer Stichprobe in sechs spezifische Rollen kategorisieren: Die Mobber (8\%), die sich durch aktives Mobbingverhalten auszeichnen: Sie sind die Anführer. Die Assistenten (7 \%) machen aktiv beim Mobbing mit, wenn ein Mobber damit angefangen hat. Die Verstärker (19\%) des Täters mobben nicht aktiv mit, geben dem Täter jedoch positives Feedback. Dies kann durch Zuschauen, Lachen, Zurufen oder ermunternde Gesten geschehen. Die Aussenseiter (24 \%) möchten sich weder auf die Seite des Täters noch des Opfers stellen. Sie lassen jedoch das Mobbing zu, indem sie sich zurückziehen und nichts machen. Schüler, die klar gegen das Mobbing sind, das Opfer trösten, sich auf dessen Seite stellen und die Anderen vom Mobbing abzubringen versuchen, werden Verteidiger (17\%) des Opfers (12\%) genannt.

Salmivalli et al. (1996) fanden gleichzeitig signifikante Geschlechtseffekte. Die Mädchen reagierten häufiger mit Rückzug und Passivität als die Jungen, sie standen häufiger für das Opfer ein und versuchten andere vom Mobben abzubringen. Die Jungen reagierten allgemein aggressiver, halfen dem Täter beim Mobben und verstärkten ihn z.B. durch Zurufen. Diese Geschlechtsunterschiede konnten in weiteren Studien repliziert werden (Salmivalli, Lappalainen \& Lagerspetz, 1998; Salmivalli \& Voeten, 2004; Schäfer \& Korn, 2004).

$\mathrm{Zu}$ möglichen Altersunterschieden bei den Zeugenreaktionen in Mobbingsituationen ist die Befundlage gering. Lindeman, Harakka und Keltikangas-Järvinen (1997) und Staub et al. (2003) fanden sinkende prosoziale Zeugenreaktionen mit zunehmendem Alter. Dies ist ein erstaunliches Ergebnis, da aggressives Verhalten (v.a. physische Aggression) mit zunehmendem Alter generell abnimmt und prosoziales Verhalten zunimmt (Coie \& Dodge, 1998; Eisenberg \& Mussen, 1989).

Ob Kinder je nach ihrer Mobbingrolle (Mobber oder Opfer) unterschiedlich reagieren, wenn sie Zeugen von Mobbing werden ohne selber betroffen zu sein, wurde bisher erst in zwei empirischen Studien untersucht. Menesini et al. (1997) fragten 6758 Kinder aus England (8-16 Jahre alt) und 1379 Kinder aus Italien (8-14 Jahre alt) nach ihren Reaktionen, wenn sie Zeugen von Mobbing würden. In beiden Ländern berichteten die Opfer häufiger, dass sie intervenieren würden als die Mobber. Und die englischen Opfer täten dies auch häufiger als die nichtinvolvierten Kinder. Bei diesem Resultat ist zu bemerken, dass die Schülerinnen und Schüler selbst Auskunft über ihr Verhalten gaben. Auch wenn die Opfer sagen, dass sie anderen Opfern helfen würden, wenn sie Zeuge einer Mobbingsituation wären, muss dies nicht heissen, dass sie tatsächlich so reagieren. Die Täter ihrerseits berichteten am seltensten, dass sie intervenieren würden. Da Mobber per definitionem aggressiver als passive Opfer und nicht-involvierte Kinder sind, kann angenommen werden, dass sie als Zeugen von Mobbingsituationen nicht nur weniger intervenieren, um das Mobbing zu stoppen, sondern sich auch aggressiver verhalten als andere Kinder, d.h. den aktuellen Mobber unterstützen würden. 
In der Mobbingforschung wird zwischen zwei Typen von Opfern unterschieden: diejenigen, die sich selten zur Wehr setzen, werden passive Opfer genannt, während Kinder, die selber aggressiv auftreten und von ihren Gleichaltrigen gemobbt werden, aggressive Opfer genannt werden. Bekannte Merkmale der Kinder in den verschiedenen Mobbingrollen lassen einige Vermutungen bezüglich Zeugenreaktionen aufkommen. So werden die passiven Opfer z.B. als ängstlich, unsicher und zurückhaltend beschrieben (Boulton \& Smith, 1994; Olweus, 1999), was zur Annahme führt, dass sie als Mobbingzeugen hilfloser reagieren als die anderen Kinder und sich vermehrt zurückziehen. Die aggressiven Opfer werden von Salmivalli und Nieminen (2002) als noch aggressiver als die Mobber beschrieben. Bei dieser Gruppe liegt die Vermutung nahe, dass sie sich als Zeugen von Mobbingsituationen auch aggressiv verhalten.

In der vorliegenden Studie werden Zeugenreaktionen von Kindergartenkindern in Abhängigkeit von Geschlecht, Alter und Mobbingrolle untersucht. Wir erwarten mehr Opfer-unterstützenes Verhalten von Seiten der Mädchen und mehr Mobber-unterstützendes Verhalten von Seiten der Mobber. Ansonsten lassen wir die Hypothesen offen, da die bisherige Forschungsgrundlage relativ gering ist. Gestützt auf dem Befund von Salmivalli und Voeten (2004), die zeigen konnten, dass das Verhalten der Gruppenmitglieder ein wichtiger Prädiktor für das Verhalten eines einzelnen Kindes ist, soll geprüft werden, wie das Verhalten der Kinder in der Gruppe mit den Zeugenreaktionen der einzelnen Kindern zusammenhängt. Wir erwarten, dass in Gruppen, in welchen viel aggressives Verhalten vorkommt mehr Mobber-unterstützendes Verhalten auftritt.

\section{Methode}

\section{St ichprobe}

Für die vorliegende Studie wurden 59 Kindergärten aus einem grösseren Projekt $^{1}$ ausgewählt. Das Auswahlkriterium war, dass es in einer Kindergartengruppe mindestens einen identifizierten Mobber oder ein Opfer (passives oder aggressives) gab. Die ursprüngliche Stichprobe ist anderweitig im Detail beschrieben (Alsaker \& Nägele, submitted 2008; Alsaker, Nägele, Valkanover \& Hauser, 2008). Die endgültige Stichprobe umfasste 968 Kinder (100\%), darunter 468 Mädchen (48.3\%) und 500 Jungen (51.7\%). Das durchschnittliche Alter der Kinder betrug 5.8 Jahre $(S D=0.57)$, die Alterspanne lag zwischen 4.8 und 7.7 Jahren.

\section{Instrumente}

Mobbingrolle. Die Mobbingrollen wurden anhand der Angaben der Kindergärtnerinnen erhoben. Das Instrument wurde bereits in einer früheren Studie zu Mobbing im Kindergarten eingesetzt (Perren \& Alsaker, 2006) und zeigte gute Übereinstimmung mit den Aussagen der Kinder zum Mobbinggeschehen. Die 
Kindergärtnerinnen waren im Vorfeld der Befragung geschult worden, um Mobbingverhalten besser zu erkennen (Alsaker \& Nägele, subm. 2008). Danach schätzten sie jedes Kind einzeln hinsichtlich seines Verhaltens in den letzten drei Monaten ein. Es wurde zwischen physischem («...das Kind wird physisch geplagt [geschlagen, getreten, gekniffen, gebissen...]»), verbalem («...das Kind wird verbal geplagt [ausgelacht, beschimpft, gehänselt...]»), gegenständlichem («...dem Kind werden Dinge versteckt oder kaputt gemacht») und indirektem Mobbing ("...das Kind wird von anderen ausgeschlossen») unterschieden. Die Häufigkeit dieser Vorkommnisse wurde jeweils entlang einer Skala von 1) nie, selten, einmal oder häufiger pro Monat, einmal pro Woche, bis 5) mehrmals pro Woche angegeben.

Kinder, die mindestens einmal pro Woche auf mindestens eine Art von anderen gemobbt wurden, selber nie oder selten aggressiv auftraten, wurden als passive Opfer bezeichnet. Kinder, die mindestens einmal pro Woche auf irgendeine Art andere Kinder mobbten, selber aber nie oder selten gemobbt wurden, wurden als Mobber kategorisiert. Kinder, die mindestens einmal pro Woche auf irgendeine Art gemobbt wurden und die mindestens einmal pro Woche andere Kinder mobbten, wurden als aggressive Opfer bezeichnet. Als nicht-involvierte Kinder galten jene, die nie oder selten gemobbt wurden und nie oder selten aggressiv auftraten. Kinder, die keiner dieser Kategorien zugeteilt werden konnten, wurden Mix-Kinder genannt. Diese Kinder waren nie öfter als einmal pro Monat in Mobbinggeschehnisse involviert. 124 Kinder wurden als Mobber (12.8\%), 63 als passive Opfer (6.5\%), 79 als aggressive Opfer (8.2\%), 479 als nicht-involvierte Kinder (49.5\%) und 194 als Mix-Kinder (20\%) kategorisiert.

Zeugenreaktionen. Die Kindergärtnerinnen gaben an, wie sich ein Kind typischerweise verhielt, wenn es Zeuge von Mobbingvorfällen wurde. Es wurden neun Reaktionsweisen aufgelistet, die auf einer 4-stufigen Skala (nie, selten, oft, fast immer) beurteilt werden mussten. Die Items wurden in Anlehnung an bereits existierenden Studien zu den Zeugenreaktionen in Mobbingsituationen entwickelt (z.B. Salmivalli et al., 1996; Sutton \& Smith, 1999). Die Skalenanalyse zur faktoriellen Struktur ergab folgende drei Skalen: Opfer-unterstützende Zeugenreaktionen (3 Items: "Das Kind versucht, das Opfer aufzumuntern oder zu trösten", "Das Kind versucht, dem Opfer zu helfen", "Das Kind setzt sich bei den anderen Kindern für das Opfer ein»; $\alpha=.93, N=850$ ), Mobber-unterstützende Zeugenreaktionen (2 Items: "Das Kind lacht und findet es lustig», "Das Kind hilft dem Täter»; $\alpha=.85, N=874$ ), Hilflose Zeugenreaktionen (2 Items: "Das Kind reagiert gar nicht», "Das Kind zieht sich zurück»; $\alpha=.55, N=875)$.

Zwei Items wurden aus statistischen Gründen aus den Analysen ausgeschlossen. Sie brachten einen zu geringen Beitrag zur internen Konsistenz, d.h. von neun erhobenen Items wurden nur sieben verwendet. Die drei gebildeten Zeugenreaktionsskalen sind mit jenen aus der Zeugenliteratur vergleichbar (vgl. z.B. Craig \& Pepler, 1997; Lindeman et al., 1997; Staub et al., 2003). 
Alter. Der Besuch des Kindergartens ist in der Schweiz fakultativ. Trotzdem besuchen 99 \% der Kinder im Kanton Bern den Kindergarten und die allermeisten unter ihnen tun dies zwei Jahre. In der vorliegenden Studie werden die Kinder, welche das erste Kindergartenjahr besuchen als jüngere Kinder und diejenigen, die das zweite Kindergartenjahr besuchen, als ältere Kinder bezeichnet.

Habituelles Verhalten in der Gruppe. Um auch Gruppenaspekte in die Analysen miteinbeziehen zu können, wurden zwei Variablen auf Gruppenebene gebildet. Die beiden Variablen sollten das habituelle Verhalten in einer Kindergruppe annähernd abbilden. Hierfür beurteilten die Kindergärtnerinnen in 23 Kindergärten (siehe Alsaker et al., 2008 für weitere Details) das allgemeine Sozialverhalten jedes einzelnen Kindes in der Kindergartengruppe (unabhängig von der Mobbingsituation in der Gruppe). Diese individuellen Werte der Kinder wurden zu einem Gruppenmittelwert verrechnet. So entstanden die Variablen $a g-$ gressive Stimmung in der Kindergruppe und positives Verhalten in Konfliktsituationen in der Kindergruppe. Die erste Variable besteht aus drei Items («Tritt, beisst, schlägt andere Kinder», "Ist Peers gegenüber aggressiv», "Zerstört eigene oder fremde Sachen»; $\alpha=.88, N=355)$. Die zweite Variable besteht aus zwei Items («Setzt sich für die Rechte anderer Kinder ein» und «Vermittelt bei Konflikten zwischen Peers»; $\alpha=.83, N=348)$.

\section{Ergebnisse}

\section{Geschlecht, Alter, Mobbingrolle und Zeugenreaktionen}

Interkorrelationen zwischen den abhängigen Variablen ergaben signifikante negative Zusammenhänge zwischen den Opfer- und den Mobber-unterstützenden Zeugenreaktionen $r_{s}=-.12(p<.01,2$-seitig, $N=842)$ und zwischen den Opferunterstützenden und den Hilflosen Zeugenreaktionen $r_{s}=-.35(p<.01,2$-seitig, $N=841$ ), jedoch keinen Zusammenhang zwischen den Mobber-unterstützenden und den Hilflosen Zeugenreaktionen $r_{s}=-.06$ (n.s., $N=860$ ).

Der Einfluss von Geschlecht, Alter und Mobbingrolle auf das Zeugenverhalten wurde anhand von drei Varianzanalysen (ANOVA) mit den drei Faktoren Geschlecht, Alter und Mobbingrolle und den jeweiligen abhängigen Variablen Opfer-unterstützende Zeugenreaktionen, Mobber-unterstützende Zeugenreaktionen und Hilflose Zeugenreaktionen überprüft. Es gab keine Interaktionen zwischen Geschlecht, Alter und Mobbingrollen in diesen Analysen. Die Ergebnisse werden jeweils für alle drei Reaktionstypen und je eine der drei unabhängigen Variablen tabellarisch dargestellt (siehe Tabellen 1-3). Es zeigte sich, dass sich die Mädchen und Jungen in den Opfer-unterstützenden und in den Mobber-unterstützenden Zeugenreaktionen signifikant voneinander unterscheiden. Bei den Hilflosen Zeugenreaktionen waren keine Geschlechtsunterschiede zu finden (Tabelle 1). Die Mädchen zeigten signifikant mehr Opfer-unterstützende Zeugenreaktionen als die Jungen (schwacher bis mittlerer Effekt nach Cohen, 
1988). Die Jungen reagierten signifikant häufiger Mobber-unterstützend als die Mädchen (schwacher bis mittlerer Effekt nach Cohen, 1988).

Tabelle 1: Signifikanztests für den Haupteffekt des Geschlechts auf die Zeugenreaktionen

\begin{tabular}{|lccccccc|}
\hline Abhängige Variable & $\mathrm{n}^{2}$ & $\mathrm{df}$ & $\mathrm{F}$ & \multicolumn{2}{c}{ Mädchen $(\mathrm{n}=402)$} & \multicolumn{2}{c|}{ Jungen $(\mathrm{n}=429)$} \\
& & & & $\mathrm{M}$ & $\mathrm{SD}$ & $\mathrm{M}$ & $\mathrm{SD}$ \\
\hline Opfer-unterstützende ZR & .039 & 1 & $32.72^{* * *}$ & 2.35 & .79 & 2.00 & .75 \\
Mobber-unterstützende ZR & .028 & 1 & $23.22^{* * *}$ & 1.49 & .62 & 1.83 & .79 \\
Hilflose ZR & .000 & 1 & 0.01 & 2.18 & .74 & 2.18 & .73 \\
\hline
\end{tabular}

Anmerkungen: $\mathrm{ZR}=$ Zeugenreaktion, ${ }^{* * *} \mathrm{p}<.001$

Die Analysen zeigten weiter, dass die älteren Kinder signifikant mehr Opferunterstützend reagierten als die jüngeren Kinder (mittlerer Effekt nach Cohen, 1988). Die jüngeren Kinder zeigten signifikant mehr Hilflose Zeugenreaktionen als ihre älteren Mitschüler. Bezüglich Mobber-unterstützenden Zeugenreaktionen konnten keine signifikanten Unterschiede gefunden werden (Tabelle 2).

Tabelle 2: Signifikanztests für den Haupteffekt des Alters auf die Zeugenreaktionen

\begin{tabular}{|lccccccc|}
\hline Abhängige Variable & $\eta^{2}$ & $\mathrm{df}$ & $\mathrm{F}$ & \multicolumn{2}{c}{ 1. Kiga $(\mathrm{n}=309)$} & \multicolumn{2}{c|}{ 2. Kiga (n=522) } \\
& & & & $\mathrm{M}$ & $\mathrm{SD}$ & $\mathrm{M}$ & $\mathrm{SD}$ \\
\hline Opfer-unterstützende ZR & .072 & 1 & $33.93^{* * *}$ & 1.89 & .72 & 2.33 & .79 \\
Mobber-unterstützende ZR & .003 & 1 & 0.78 & 1.59 & .70 & 1.71 & .70 \\
Hilflose ZR & .012 & 1 & $5.12^{* *}$ & 2.29 & .74 & 2.11 & .73 \\
\hline
\end{tabular}

Anmerkungen: $\mathrm{ZR}=$ Zeugenreaktionen, Kiga $=$ Kindergartenjahr, ${ }^{* *} \mathrm{p}<.01,{ }^{* * *} \mathrm{p}<.001$

Die univariaten Signifikanztests für den Haupteffekt «Mobbingrolle» zeigten, dass sich die Mobber, passiven Opfer, aggressiven Opfer, nicht-involvierten Kinder und die Mix-Kinder signifikant in den Mobber-unterstützenden, in den Opfer-unterstützenden und in den Hilflosen Zeugenreaktionen unterschieden (schwache bis starke Effekte nach Cohen, 1988; Tabelle 3).

Die nicht-involvierten Kinder zeigten signifikant mehr Opfer-unterstützende Zeugenreaktionen als die aggressiven Opfer, die Mobber und die passiven Opfer. Die Mobber und aggressiven Opfer zeigten signifikant mehr Mobber-unterstützende Zeugenreaktionen als die nicht-involvierten Kinder, die passiven Opfer und die Mix-Kinder. Die passiven Opfer zeigten signifikant mehr Hilflose Zeugenreaktionen als alle anderen Kinder. 
Tabelle 3: Signifikanztests für den Haupteffekt Mobbingrolle

\begin{tabular}{|lcccl|}
\hline Abhängige Variable & $\eta^{2}$ & df & F & Post-Hoc Test (Tukey-HSD) \\
\hline Opfer-unterstützende ZR & .018 & 4 & $3.62^{* *}$ & $\mathrm{nI}>\mathrm{AO}(\mathrm{p}<.01), \mathrm{M}(\mathrm{p}<.05), \mathrm{PO}(\mathrm{p}<.1)$ \\
& & & & \\
\hline Mobber-unterstützende ZR & .325 & 4 & $97.63^{* * *}$ & $\mathrm{M}>\mathrm{nI}(\mathrm{p}<.001), \mathrm{PO}(\mathrm{p}<.001)$, \\
& & & & $\mathrm{Mix}(\mathrm{p}<.001) ;$ \\
& & & & $\mathrm{AO}>\mathrm{nI}(\mathrm{p}<.001), \mathrm{PO}(\mathrm{p}<.001)$, \\
& & & $\mathrm{Mix}(\mathrm{p}<.001)$
\end{tabular}

Anmerkungen: $\mathrm{ZR}=$ Zeugenreaktionen; $\mathrm{M}=$ Mobber; $\mathrm{PO}=$ passive Opfer, $\mathrm{AO}=$ aggressive Opfer, $\mathrm{nI}=$ nicht involvierte Kinder; Mix $=$ Kinder in der Mix-Kategorie, ${ }^{* *} \mathrm{p}<.01,{ }^{* * *} \mathrm{p}<.001$

Habituelles Verhalten in der Gruppe und Zeugenreaktionen Der Zusammenhang zwischen dem habituellen Verhalten der Kinder in der Kindergartengruppe und den Zeugenreaktionen wurde für die Teilstichprobe von 373 Kindern untersucht und wegen stärkerer Abweichung von der Normalverteilung bei den involvierten Variablen mit einer Rangkorrelation nach Spearman geprüft (Tabelle 4).

Tabelle 4: Rangkorrelation nach Spearman zwischen den Gruppenvariablen und den Zeugenreaktionen

\begin{tabular}{|c|c|c|c|c|c|c|}
\hline & & 1 & 2 & 3 & 4 & 5 \\
\hline 1 & Aggressive Stimmung in der Kindergruppe & 1 & $-.27^{* *}$ & $-.13^{* *}$ & $.50^{* *}$ & $-.12^{* *}$ \\
\hline 2 & $\begin{array}{l}\text { Positives Verhalten in Konfliktsituationen in der } \\
\text { Kindergruppe }\end{array}$ & & 1 & $.76^{* *}$ & $-.21^{* *}$ & $-.33^{* *}$ \\
\hline 3 & Opfer-unterstützende ZR & & & 1 & $-.14^{* *}$ & $-.35^{* *}$ \\
\hline 4 & Mobber-unterstützende ZR & & & & 1 & $-.13^{* *}$ \\
\hline 5 & Hilflose ZR & & & & & 1 \\
\hline
\end{tabular}

Anmerkungen: $\mathrm{ZR}$ = Zeugenreaktionen; paarweiser Fallausschluss; die Stichprobengrösse variiert von $\mathrm{N}=$ 345 bis $\mathrm{N}=371 ;^{* *} \mathrm{p}<.01$ (einseitige Testung)

Je mehr aggressives Verhalten in einer Kindergartengruppe gezeigt wurde, umso mehr Mobber-unterstützende Zeugenreaktionen wurden gezeigt $\left(r_{s}=.50, p<\right.$ $.01)$. Die aggressive Stimmung in der Gruppe korrelierte zudem negativ mit den Opfer-unterstützenden und den Hilflosen Zeugenreaktionen $\left(r_{s}=-.13, p<.01\right.$ resp. $\left.r_{s}=-.12, p<.01\right)$. In Gruppen, in welchen viel positives Verhalten in Konfliktsituationen gezeigt wurde, wurden die Mobbingopfer von den Zeugen auch stark unterstützt $\left(r_{s}=.76, p<.01\right)$. Das positive Verhalten in Konfliktsituationen korrelierte negativ mit den Mobber-unterstützenden und den Hilflosen Zeugenreaktionen $r_{s}=-.21, p<.01$ resp. $\left.r_{s}=-.33, p<.01\right)$. 


\section{Diskussion}

Im Zentrum der vorliegenden Studie stand die Frage, wie Kindergartenkinder reagieren, wenn sie Zeugen von Mobbing werden. Es wurde untersucht, ob Geschlecht, Alter und Mobbingrolle einen Einfluss auf die Art der gezeigten Zeugenreaktionen hatten. Zudem wurde der Zusammenhang zwischen dem vorherrschenden sozialen Verhalten in der Kindergartengruppe und den Zeugenreaktionen geprüft.

\section{Geschlecht, Alter, Mobbingrolle und Zeugenreaktionen}

Aus den vorliegenden Ergebnissen wurde ersichtlich, dass Jungen als Mobbingzeugen signifikant häufiger die Mobber unterstützten und beim Mobben mitmachten, während die Mädchen häufiger den Opfern halfen und sich für diese einsetzten. Diese Resultate bestätigen Befunde aus früheren Studien zu den Zeugenreaktionen bei Mobbingvorfällen (Salmivalli et al., 1996; Salmivalli et al., 1998; Salmivalli \& Voeten, 2004; Schäfer \& Korn, 2004). Bezüglich der hilflosen Zeugenreaktionen waren keine Geschlechtsunterschiede festzustellen. Mädchen und Jungen entfernten sich gleich oft von Mobbingsituationen und schauten weg. Es ist anzunehmen, dass sich die Kindergartenkinder (5- bis 7-jährig) aus den Problemsituationen zurückzogen, weil sie sich überfordert fühlten und nicht wussten, was zu tun war, oder auch, weil sie Angst hatten, selbst zum Opfer zu werden, wenn sie intervenieren würden (Slee, 1993). Wie bereits frühere Studien zeigten, intervenieren viele Kinder nicht, obwohl sie in Mobbingsituationen anwesend sind (Hawkins et al., 2001). Somit verhalten sich die Kindergartenkinder in unserer Studie nicht anders als ältere Kinder.

Wir konnten die Befunde von Lindeman et al. (1997) und Staub et al. (2003), welche besagten, dass die prosozialen Zeugenreaktionen mit zunehmendem Alter abnehmen, nicht bestätigen. In unserer Studie reagierten die jüngeren Kindergartenkinder in Mobbingsituationen hilfloser als die Älteren und die Älteren zeigten signifikant mehr Opfer-unterstützende Zeugenreaktionen als die Jüngeren. Dies korrespondiert mit dem vielfach dokumentierten Befund, dass prosoziales Verhalten mit dem Alter generell zunimmt (vgl. z.B. Eisenberg \& Mussen, 1989). Da in der vorliegenden Studie Querschnittdaten erhoben wurden, können wir keine Aussagen darüber machen, ob bei Kindergartenkindern mit zunehmendem Alter die hilflosen Reaktionen zugunsten der angemessenen zurückgehen. Die älteren Kinder scheinen jedoch mehr Strategien zur Verfügung zu haben, um das Opfer zu unterstützen und somit die eigene (mögliche) Hilflosigkeit überwinden zu können. Weiter ist es auch möglich, dass die älteren Kinder klarere Vorstellungen davon haben, dass Mobbing nicht in Ordnung ist. Bezüglich Mobber-unterstützenden Zeugenreaktionen waren keine Altersunterschiede auszumachen, obwohl auch hier eine gewisse Abnahme zu erwarten wäre, da aggressives Verhalten generell mit dem Alter abnimmt (Tremblay et al., 2004). 
Die Kindergartenkinder verhielten sich je nach eigener Rolle im Mobbinggeschehen als Mobbingzeugen unterschiedlich. Die nicht-involvierten Kinder zeigten mehr Opfer-unterstützende Reaktionen als die aggressiven Opfer, die Mobber und die passiven Opfer. Die Mobber und die aggressiven Opfer zeigten mehr Mobber-unterstützende Zeugenreaktionen als alle anderen Kinder. Die passiven Opfer reagierten signifikant häufiger hilflos als alle anderen Kinder. Diese Ergebnisse zeigen ein weiteres $\mathrm{Mal}$, dass die Charakteristika der Kinder in den verschiedenen Mobbingrollen sehr unterschiedlich sind (Alsaker \& Nägele, subm. 2008) und sich auch in den Reaktionen als Mobbingzeugen niederschlagen (Bernstein, Hughes \& Watson 1997; Menesini et al., 1997). Die ängstlichen, unsicheren und zurückhaltenden passiven Opfer (Boulton \& Smith, 1994; Olweus, 1999) verhalten sich auch als Mobbingzeugen unsicher und hilflos. Als Opfer laufen sie natürlich besondere Gefahr, in solchen beobachteten Mobbingsituationen selbst zum Opfer zu werden, falls sie eingreifen würden. Die Mobber und die aggressiven Opfer hingegen (die sich allgemein dominant, aggressiv und impulsiv verhalten; Olweus, 1978, 1999; Salmivalli \& Nieminen, 2002) reagieren auch als Mobbingzeugen auf antisoziale Art, indem sie die Mobber unterstützen. Während bei Menesini und Kollegen (1997) die Kinder selber Auskunft über ihr Verhalten gaben und deshalb Effekte sozialer Erwünschtheit nicht auszuschliessen sind, waren in der vorliegenden Studie die Kindergärtnerinnen die auskunftsgebenden Personen zum Verhalten der Kinder.

\section{Habituelles Verhalten in der Gruppe und Zeugenreaktionen}

Der Einbezug aggregierter Gruppenvariablen in die Analysen wurde unternommen um Gruppenaspekte systematisch einzubeziehen. Die Ergebnisse zeigten, dass eine aggressive Stimmung in der Kindergartengruppe, d.h. viel aggressives Verhalten in der Gruppe, mit mehr Mobber-unterstützenden Zeugenreaktionen einherging. Positives Verhalten in Konfliktsituationen in der Kindergartengruppe ging mit mehr Opfer-unterstützendem und weniger Mobber-unterstüzendem Verhalten einher. Die Ergebnisse sind korrelativer Art und es bleibt unklar, ob z.B. die generell aggressive Stimmung dazu führte, dass mehr Mobber-unterstützende Zeugenreaktionen gezeigt wurden oder ob die Korrelation nur die Tatsache reflektiert, dass es in diesen Kindergartengruppen viele aggressive Kinder gab, die einander in ihren aggressiven Handlungen unterstützen. Wie bereits erwähnt, lernen Kinder viele Verhaltensweisen durch Beobachten und Nachahmen Anderer (Bandura, 1977). Daraus lässt sich ableiten, dass ein Kind desto eher aggressives Verhalten zeigt, je mehr aggressive Modelle sich in seiner Kinergartengruppe befinden. Je länger das Mobbing schon andauert, umso selbstverständlicher und normaler wird dieses negative Verhalten für die Kinder, sodass beim einzelnen Kind die Hemmung, beim Mobbing mitzuhelfen, nachlässt (Alsaker, 2003; Olweus, 2002). Ein weiterer Gruppenmechanismus, welcher das Resultat zur aggressiven Stimmung in der Kindergartengruppe erklären kann, ist die früher genannte Verantwortungsdiffusion. Je stärker die ag- 
gressive Stimmung in einer Gruppe ist, d.h. je mehr Kinder aggressives Verhalten zeigen, desto weniger fühlen sich die einzelnen Akteure für ihre negativen Handlungen verantwortlich; es haben ja schliesslich viele mitgemacht. Kritisch gilt anzumerken, dass die generelle Stimmung oder das habituelle Verhalten in der Gruppe in unserer Studie durch Indikatoren für individuelles Sozialverhalten erfasst wurde. Es stellt lediglich eine Approximation einer Gruppenvariable dar. Trotz dieser Einschränkung zeigen die signifikanten Resultate, dass die Gruppe als Ganzes in späteren Studien mit berücksichtigt werden sollte, da sie einen Einfluss auf das Verhalten einzelner Kinder haben kann.

\section{Implikationen für die Praxis}

Unsere Ergebnisse zeigen klar, dass Mobbing mit allen Kindern in einer Gruppe angegangen werden sollte. Frühere Studien belegten, dass das Verhalten der Mobbingzeugen den Beginn und die Kontinuität der Mobbing-Interaktion beeinflusst (O'Connell et al., 1999). Die Tatsache, dass die meisten Kinder dem Mobbing gegenüber negativ eingestellt sind (Menesini et al., 1997; Rigby \& Slee, 1991), aber trotzdem nicht zugunsten der Opfer intervenieren oder sogar zusammen mit den Mobbern «mitmobben» (Craig \& Pepler, 1995, 1997; Hawkins et al., 2001; O’Connell et al., 1999) zeigt, wie wichtig die grosse Gruppe der Mobbingzeugen in der Prävention ist. Deshalb wird in zahlreichen Präventionsprogrammen mit dem ganzen System Klasse/Kindergruppe gearbeitet. Dies ist beispielsweise im "Berner Programm gegen Gewalt in Kindergarten und Schule: Be-Prox» der Fall (Alsaker, 2003; 2004; Alsaker et al., 2008). Die Mobbingzeugen sind in diesem Programm eine wichtige Zielgruppe. Auch andere Präventionsprogramme wurden entwickelt, in welchen die Peers als Mobbingzeugen eine zentrale Stellung einnehmen. Zu nennen sind beispielsweise das finnische «Anti-Bullying-Programm» (Salmivalli, 1999; Salmivalli et al., 2004), das norwegische "Anti-Bullying-Program» von Olweus (1991), das belgische Programm von Stevens, Van Oost und De Bourdeaudhuij (2000) und das englische «Sheffield Anti-Bullying Program» (Smith \& Sharp, 1994).

In der vorliegenden Studie halfen die älteren Kinder den Opfern öfter als die jüngeren, welche mehr hilflose Zeugenreaktionen zeigten. Wie bereits von anderen Autoren vorgeschlagen wurde, könnte es sinnvoll sein, einigen Kindern in der Kindergarten- oder Schulklasse einen besonderen Stellenwert einzuräumen (Mentoren-Programme; Peer-helpers etc.; Cowie, 2000; Pepler, Smith \& Rigby, 2004; Salmivalli, 1999). So könnte z.B. eine Art «Paten/Götti-System» eingeführt werden. Dabei würde jedem jüngeren Kind ein älteres zur Seite gestellt, welches die Aufgabe erhielte, dem jüngeren zu helfen, falls es Schwierigkeiten hätte (egal welcher Art). Dieses System könnte zusätzlich zu einer besseren Stimmung in der Gruppe führen, was wiederum die Zeugenreaktionen beeinflussen sollte.

Unsere Resultate zeigten, dass bereits im Kindergarten die Mobber in ihren Handlungen oft von Mobbingzeugen unterstützt werden. Ein wichtiger Schritt in der Prävention ist deshalb, dem Mobber das Publikum wegzunehmen (Salmi- 
valli et al., 2004). Wird ein mobbendes Kind in seinen Handlungen immer wieder verstärkt und angefeuert, wird es immer schwieriger, sein Verhalten zu stoppen. Um zu erreichen, dass die Mobbingzeugen dieses Verhalten nicht weiter verstärken, müssen die Kinder für die Mobbingthematik sensibilisiert werden, und es muss auch über die Gruppenmechanismen im Mobbing gesprochen werden (Salmivalli et al., 2004). Den Kindern muss klar werden, dass jede einzelne Person dafür verantwortlich ist, ob Mobbing in der Klasse vorkommt oder nicht (Alsaker, 2003) und dass weder das Mobben, das Mitmobben, noch das Zuschauen oder Weggehen angemessene, tolerierbare Reaktionen sind. Den Kindern muss geholfen werden, alternative Verhaltensweisen zu finden, wie auf Mobbing reagiert werden kann. Anhand von Rollenspielen könnten diese z.B. gezeigt und geübt werden (Alsaker, 2003; Salmivalli et al., 2004; Stevens et al., 2000). Das alleinige Reden darüber, wie man sich als Zeuge verhalten sollte, reicht bei Kindern meistens nicht. Sie brauchen verbindliche Regeln (Alsaker, 2003; Pepler et al., 2004; Salmivalli et al., 2004; Stevens et al., 2000), die z.B. in der Kindergartengruppe gemeinsam vereinbart werden. Solche Regeln können Hinweise auf angemessenes Intervenieren gegen Mobbing beinhalten. Der Befund in der vorliegenden Studie, dass die sogenannten nicht-involvierten Kinder mehr Opfer-unterstützende Zeugenreaktionen zeigten als die anderen Kinder, macht deutlich, dass diese Kinder in der Mobbingprävention eine grosse Ressource darstellen. Ein Präventionsziel wäre deshalb auch, die Anzahl jener Kinder zu erhöhen, die beim Mobbing aktiv zugunsten des Opfers intervenieren. Um effektive Prävention zu betreiben, sollte diese früh, intensiv und langfristig verankert stattfinden.

\section{Einschränkungen und Ausblick}

In der vorliegenden Studie nahmen 59 Kindergärten teil. Die Auskünfte zum Verhalten der Kinder erhielten wir von den Kindergärtnerinnen. Da diese zu mehreren Kindern Aussagen machen mussten, ergibt sich das Problem der Abhängigkeit der Daten. Es ist nicht auszuschliessen, dass sich die Kindergärtnerinnen bei ihren Bewertungen von vorherrschenden Stereotypen leiten liessen (d.h. den Mädchen wurde möglicherweise aufgrund eines Stereotyps mehr prosoziales Verhalten zugeschrieben als den Jungen). Zudem ist die Nicht-Unabhängigkeit der Daten auch aufgrund der Verwendung einer Klumpenstichprobe gegeben. Um für Effekte auf Gruppenebene zu kontrollieren, müssten daher bei weiteren Untersuchungen Mehrebenen-Analysen durchgeführt werden. Dies bedeutet, dass die Stichprobengrösse resp. die Anzahl Gruppen und Anzahl der einzelnen Beobachtungen pro Gruppe sowie das verwendete Modell zur Parameterschätzung bereits vor Untersuchungsbeginn anhand geeigneter Verfahren festgelegt werden sollten (z.B. Bickel, 2007).

Eine weitere Einschränkung der vorliegenden Studie ist, dass die Kindergärtnerinnen nur befragt wurden, wie jedes Kind reagierte, wenn es einen Mobbingvorfall beobachtete, was einen beobachtbaren Mobbingvorfall voraussetzte. Da 
beobachtbare Mobbingvorfälle im Kindergarten meist physischer (oder verbaler) Natur sind, ist auch davon auszugehen, dass Jungen, die mehr physische Aggression zeigen als Mädchen (Coie \& Dodge, 1998), als Mobbingzeugen häufiger physisch aggressiv reagieren und den Mobber assistieren als Mädchen. Würde z.B. untersucht werden, wie Mobbingzeugen reagieren, wenn der Mobber relationales aggressives Verhalten zeigt (z.B. ein anderes Kind ausschliesst), wären es vielleicht die Mädchen, welche die Mobber mehr unterstützten als die Jungen.

Da Mobbing oft im Versteckten passiert, wäre es sinnvoll, die Einschätzungen der Kindergärtnerinnen mit weiteren Methoden zu ergänzen. Eine geeignete Erhebungsmethode wäre z.B. die naturalistische Beobachtung der Kinder im Kindergarten (mit Videokameras; Craig \& Pepler, 1995, 1997). Da Beobachtungen nur punktuell vorgenommen werden können und die Gefahr besteht, dass die Beobachtung das Verhalten der Beobachteten verändert, sollte auf die Einschätzungen der Kindergärtnerinnen trotzdem nicht verzichtet werden. $\mathrm{Zu}-$ dem beziehen sich die hier berichteten Einschätzungen der Kindergärtnerinnen zu den Zeugenreaktionen der Kinder auf längerfristige Beobachtungen.

Die vorliegende Studie zeigte, dass jedes Kind in der Gruppe eine Rolle im Mobbingprozess einnimmt. Für nachfolgende Studien und für die weitere Entwicklung von Präventionsprogrammen ist es deshalb von grosser Bedeutung, Mobbing nicht nur als Mobber-Opfer-Dyade, sondern als Gruppenphänomen zu betrachten. Jede/r Einzelne trägt einen Teil dazu bei, ob Mobbing entsteht und aufrechterhalten wird.

\section{Anmerkung}

1 Die vorliegende Studie ist Teil der Längsschnittstudie «Mobbing im Kindergarten und in der Schule: Entstehung und Prävention». Die Studie wurde vom Schweizerischen Nationalfonds zur Förderung der wissenschaftlichen Forschung unterstützt (NFP 52; Projektnummer 4052-69011).

\section{References}

Alsaker, F. D. (2003). Quälgeister und ihre Opfer. Bern: Hans Huber Verlag.

Alsaker, F. D. (2004). Bernese programme against victimisation in kindergarten and elementary school. In P. K. Smith, D. Pepler \& K. Rigby (Hrsg.), Bullying in schools. How successful can interventions be? (S. 289-306). Cambridge: Cambridge University Press.

Alsaker, F. D. \& Nägele, C. (submitted, Dezember 2008). Vulnerability to victimization in kindergarten: Need for a differentiation between passive and aggressive victims. MerrilPalmer Quarterly.

Alsaker, F. D., Nägele, C., Valkanover, S. \& Hauser, D. (2008). Pathways to victimization and a multisetting intervention. project documentation. Universität Bern.

Atlas, R. S. \& Pepler, D. J. (1998). Observations of bullying in the classroom. The Journal of Educational Research, 92, 86-99.

Bandura, A. (1977). Social learning theory. Englewood Cliffs, N.J: Prentice-Hall.

Bernstein, J. Y., Hughes, L. J. \& Watson, M. W. (1997). The beliefs and behaviors of bullies, victims and problem-solvers. Paper presented at the Meetings of the Society for Research in Child Development, Washington, DC. 
Bickel, R. (2007). Multilevel analysis for applied research. It's just regression! New York: The Guilford Press.

Boulton, M. J. \& Smith, P. K. (1994). Bully/victim problems in middle-school children: stability, self-perceived competence, peer perceptions and peer acceptance. British Journal of Developmental Psychology, 12, 315-329.

Cohen, J. (1988). Statistical Power analysis for the behavioral science. Hillsdale, NJ: Erlbaum.

Coie, J. D. \& Dodge, K. A. (1998). Aggression and antisocial behavior. In W. Damon \& N. Eisenberg (Hrsg.), Handbook of child psychology, Vol. 3. Social, emotional, and personality development (S. 779-862). New York: Wiley.

Cowie, H. (2000). Bystanding or Standing by: Gender issues in coping with bullying in english schools. Aggressive Behavior, 26, 85-97.

Craig, W. M. \& Pepler, D. J. (1995). Peer processes in bullying and victimization: an observational study. Exceptionality Education Canada, 5, 81-95.

Craig, W. M. \& Pepler, D. J. (1997). Observations of bullying and victimization in the school yard. Canadian Journal of School Psychology, 13, 41-60.

Darley, J. M. \& Latané, B. (1968). Bystander intervention in emergencies: diffusion of responsibility. Journal of Personality and Social Psychology, 8, 377-383.

Ditton, H. (1998). Mehrebenenanalyse. Grundlagen und Anwendungen des Hierarchisch Linearen Modells. Weinheim, München: Juventa Verlag.

Eisenberg, N. \& Mussen, P. H. (1989). The roots of prosocial behavior in children. Cambridge: Cambridge University Press.

Gutzwiller-Helfenfinger, E. \& Alsaker, F. D. (2005). Who will go for help? Children as witnesses of victimization episodes. Poster presented at the Biennial Meeting of the Society for Research in Child Development, Atlanta, Georgia.

Gutzwiller-Helfenfinger, E. \& Alsaker, F. D. (2007). Participant roles and beyond-When kindergarten children witness victimization. Poster presented at the Biennial Meeting of the Society for Research in Child Development, Boston, Massachusetts (USA).

Hawker, D. S. J. \& Boulton, M. J. (2000). Twenty years' research on peer victimization and psychosocial maladjustment: a meta-analytic review of cross-sectional studies. Journal of Child Psychology and Psychiatry, 41, 441-455.

Hawkins, D. L., Pepler, D. J. \& Craig, W. M. (2001). Naturalistic observations of peer interventions in bullying. Social Development, 10, 512-527.

Latané, B. \& Darley, J. M. (1968). Group inhibition of bystander intervention in emergencies. Journal of Personality and Social Psychology, 10, 215-221.

Lindeman, M., Harakka, T. \& Keltikangas-Järvinen, L. (1997). Age and gender differences in adolescents' reactions to conflict situations: aggression, prosociality, and withdrawal. Journal of Youth and Adolescence, 26, 339-351.

Menesini, E., Eslea, M., Smith, P. K., Genta, M. L., Giannetti, E., Fonzi, A. \& Costabile, A. (1997). Cross-national comparison of children's attitudes toward bully/victim problems in school. Aggressive Behavior, 23, 245-257.

O’Connell, P., Pepler, D. \& Craig, W. (1999). Peer involvement in bullying: insights and challenges for intervention. Journal of Adolescence, 22, 437-452.

Olweus, D. (1978). Aggression in the schools: bullies and whipping boys. Washington, DC: Hempisphere.

Olweus, D. (1991). Bully/victim problems among schoolchildren: basic facts and effects of a school based intervention programm. In K. Rubin \& D. J. Pepler (Hrsg.), The development and treatment of childhood aggression (S. 411-448). Hillsdale, N. J.: Erlbaum.

Olweus, D. (1999). Sweden. In P. K. Smith, Y. Morita, J. Junger-Tas, D. Olweus, R. Catalano \& P. Slee (Hrsg.), The nature of school bullying. A cross-national perspective (S. 7-27). London: Routledge.

Olweus, D. (2002). Gewalt in der Schule. Was Lehrpersonen und Eltern wissen sollten - und tun können (3. Aufl.). Bern, Göttingen, Toronto, Seattle: Hans Huber Verlag. 
Pepler, D., Smith, P. K. \& Rigby, K. (2004). Looking back and looking forward: implications for making interventions work effectively. In P. K. Smith, D. Pepler \& K. Rigby (Hrsg.), Bullying in schools. How successful can interventions be? (S. 307-324). Cambridge: Cambridge University Press.

Perren, S. \& Alsaker, F. D. (2006). Social behavior and peer relationships of victims, bully-victims, and bullies in kindergarten. Journal of Child Psychology and Psychiatry, 47, 45-57.

Rigby, K. \& Slee, P. T. (1991). Bullying among Australian school children: reported behavior and attitudes toward victims. The Journal of Social Psychology, 131, 615-627.

Salmivalli, C. (1999). Participant role approach to school bullying: implications for interventions. Journal of Adolescence, 22, 453-459.

Salmivalli, C., Kaukiainen, A., Voeten, M. \& Sinisammal, M. (2004). Targeting the group as a whole: the Finnish anti-bullying intervention. In P. K. Smith, D. Pepler \& K. Rigby (Hrsg.), Bullying in schools. How successful can interventions be? (S. 241-273). Cambridge: Cambridge University Press.

Salmivalli, C., Lagerspetz, K., Björkqvist, K., Österman, K., \& Kaukiainen A. (1996). Bullying as a group process: participant roles and their relations to social status within the group. Aggressive Behavior, 22, 1-15.

Salmivalli, C., Lappalainen, M. \& Lagerspetz, K. M. J. (1998). Stability and change of behavior in connection with bullying in schools: A two-year follow-up. Aggressive Behavior, 24, 205-218.

Salmivalli, C. \& Nieminen, E. (2002). Proactive and reactive aggression among school bullies, victims, and bully-victims. Aggressive Behavior, 28, 30-44.

Salmivalli, C. \& Voeten, M. (2004). Connections between attitudes, group norms, and behaviour in bullying situations. International Journal of Behavioral Development, 28, 246-258.

Schäfer, M. (1996). Aggression unter Schülern. Eine Bestandesaufnahme über das Schikanieren in der Schule am Beispiel der 6. und 8. Klassenstufe. Report Psychologie, 21, 700-711.

Schäfer, M. \& Korn, S. (2004). Bullying als Gruppenphänomen: Eine Adaption des «Participant Role»-Ansatzes. Zeitschrift für Entwicklungspsychologie und Pädagogische Psychologie, 36, 19-29.

Silbereisen, R. K. (1998). Soziale Kognition: Entwicklung von sozialem Wissen und Verstehen. In R. Oerter \& L. Montada (Hrsg.), Entwicklungspsychologie (S. 823-861). Weinheim: Beltz Verlag.

Slee, P. T. (1993). Bullying: a preliminary investigation of its nature and the effects of social cognition. Early Child Development and Care, 87, 47-57.

Smith, P. K. \& Sharp, S. (1994) (Hrsg.) School bullying: insights and perspectives. London: Routledge.

Staub, E., Fellner, D., Berry, J. \& Morange, K. (2003). Passive and active bystandership across grades in response to students bullying other students. In E. Staub (Ed.), The psychology of good and evil: Why children, adults, and groups help and harm others (S. 240-243). New York: Cambridge University Press.

Stevens, V., Van Oost, P., \& De Bourdeaudhuij, I. (2000). The effects of an anti-bullying intervention programme on peers' attitudes and behaviour. Journal of Adolescence, 23, 21-34.

Sutton, J., \& Smith, P. K. (1999). Bullying as a group process: an adaption of the participant role approach. Aggressive Behavior, 25, 97-111.

Tremblay, R. E., Nagin, D. S., Séguin, J. R., Zoccolillo, M., Zelazo, P. D., Boivin, M. et al. (2004). Physical aggression during early childhood: Trajectories and predictors. Pediatrics, $114,43-50$.

Schlagworte: Gewalt in der Schule, Mobbing, Mobbingzeugen, Mobbingprävention 


\section{Enfants témoins de harcèlement à l'école enfantine}

\section{Résumé}

Cette étude examine les comportements d'enfants témoins de harcèlement. Elle est réalisée avec 968 enfants provenant de 59 écoles enfantines dans lesquelles nous avions identifié des problèmes de harcèlement. Les résultats révèlent que le genre et l'âge jouent un rôle significatif dans le type de comportements des témoins, à savoir s'ils aident les agresseurs, apportent leur soutien aux victimes ou encore assistent impuissants aux évènements. Les comportements des témoins varient aussi suivant leur rôle habituel (agresseurs ou victimes) dans les problèmes de harcèlement dans le groupe. Les agresseurs apportent plus souvent leur aide aux auteurs d'agressions, les enfants habituellement pas impliqués dans le harcèlement apportent leur soutien aux victimes et les victimes montrent des réactions d'impuissance. Le comportement habituel caractéristique du groupe des enfants dans la classe est aussi associé aux réactions d'un enfant témoin singulier: Un niveau d'agression élevé dans la classe est lié à plus d'aide aux agresseurs, alors que nous trouvons plus de comportements de soutien aux victimes dans les classes caractérisées par des comportement positifs de résolution de conflits. Le rôle des témoins dans le cadre de la prévention au harcèlement est discuté.

Mots clés: Violence à l'école enfantine, harcèlement, comportement des témoins, prévention du harcèlement

\section{I bambini dell'asilo testimoni di mobbing}

\section{Riassunto}

In questo articolo viene studiato il comportamento dei bambini testimoni di mobbing. Hanno preso parte a questa inchiesta 968 bambini provenienti da 59 asili nei quali abbiamo rilevato dei problemi di mobbing. Le analisi indicano che il sesso e l'età rivestono un ruolo significativo per il comportamento dei testimoni, sia che aiutino gli aggressori, apportino il loro sostegno alle vittime oppure assistano impotenti agli avvenimenti. I comportamenti dei testimoni variano anche in funzione del loro ruolo abituale (aggressore o vittima) nei problemi di mobbing nel gruppo. Gli aggressori portano più sovente il loro sostegno agli autori di aggressioni, i bambini che di solito non sono coinvolti dal mobbing portano il loro sostegno alle vittime, e le vittime mostrano delle reazioni di impotenza. Il comportamento abituale dei bambini nella classe è anche associato alle reazioni dei testimoni. Un alto livello di aggressioni nella classe è associato ad un sostegno più grande agli aggressori, mentre riscontriamo più comportamenti di sostegno alle vittime nelle classi caratterizzate da comportamenti positivi di risoluzione dei conflitti. Infine discuteremo il ruolo dei testimoni nell'ambito della prevenzione del mobbing.

Parole chiave: violenza a scuola, mobbing, comportamento dei testimoni, prevenzione del mobbing 


\title{
Kindergarten children witnessing victimization
}

\begin{abstract}
The present study investigates the reactions children show when witnessing victimization. It was carried out with 968 children from 59 kindergartens, in which victimization occurred. Effects of age and gender are found on both behaviour supporting the bully and behaviour supporting the victim, as well as helpless behaviour. Moreover, witnessing behaviour varies as a function of bully/victim role: Bullies display more behaviour supporting the (actual) bully; children usually not involved in bullying show more behaviour supporting the victim; and passive victims exhibit more helplessness. Behaviour in the classroom is also related to witnessing reactions: High levels of aggression in the classroom are associated with more reactions supporting the bully, whereas more reactions supporting the victim are found in groups with higher levels of positive conflict solving. The role of the witnesses is discussed within the framework of bullying prevention.
\end{abstract}

Key words: Violence in kindergarten, bullying, witnessing victimization, bullying prevention 